\title{
7. Generalbericht
}

\author{
Von Alexander Graser
}

1. Die Zielsetzung des vorliegenden Beitrags 488

2. Das Potential des Projekts 488

2.1. Der bisherige Projektverlauf 488

2.2. Die Attraktivität des Projekts und ihre Gründe $\quad 489$

2.2.1. Verschiedenheit der betrachteten Regionen 489

2.2.2. Behindertenrecht also Lackmustest der Sozialstaatlichkeit $\quad 490$

2.3. Zur Ambitioniertheit des Projekts 491

2.3.1. Vielzahl der einbezogenen Länder 491

2.3.2. Verschiedenheit der betrachteten Regionen $\quad 492$

2.3.3. Breite des Themas 492

2.3.4. Komplexität des Themas 492

3. Der Workshop im Kontext der vorangegangenen Tagungen 493

3.1. Zielsetzungen des Workshops im Lichte der vorangegangenen

Projektschritte

3.2. Ausleuchten des „kulturwissenschaftlichen Hintergrundes“ “ 494

3.3. Brückenbau ausgehend vom Boden der Rechts- und

Verwaltungswissenschaft

3.3.1. Vom materiellen Recht ausgehende Erwägungen 495

3.3.2. Vom prozeduralen Recht ausgehende Erwägungen $\quad 496$

3.3.3. Verwaltungsstrukturen und Zivilgesellschaft 497

3.4. Demographischer Wandel als Querschnittsthema 498

3.4.1. Zunahme von Behinderungen aufgrund steigender Lebenserwartung

3.4.2. Steigerung der Lebenserwartung für Menschen mit Behinderung

4. Überlegungen zur Fortsetzung des Projekts 499

4.1. Bisherige Erträge 499

4.2. Ausrichtung weiterer Schritte $\quad 500$

4.2.1. Ausgleich bestehender Asymmetrien $\quad 501$

4.2.2. Exemplarische Fokussierung 501 


\section{Die Zielsetzung des vorliegenden Beitrags}

Der vorliegende Generalbericht versucht ein Resümee des Workshops, stellt ihn in Zusammenhang mit den vorangegangenen Tagungen und erörtert die Perspektiven einer Fortsetzung des Projekts. Eine Zusammenfassung der einzelnen Beiträge ist dagegen nicht Ziel dieses Berichts. Eine vorläufige und knappere Fassung der hier entfalteten Gedanken wurde bereits während der Tagung vorgestellt, und zwar zu Beginn der Schlussdiskussion, deren Inhalt im Folgenden so weit wie möglich berücksichtigt wurde.

\section{Das Potential des Projekts}

Behindertenpolitik und -recht in Asien, Europa und darüber hinaus vielleicht auch noch in weiteren Regionen der Welt zu vermessen, ist ein faszinierendes Vorhaben, und zugleich auch ein höchst ambitioniertes. In diesem einleitenden Abschnitt wird zunächst der bisherige Projektverlauf knapp skizziert. Sodann soll substantiiert werden, was die besondere Attraktivität und Ambitioniertheit des Projekts ausmacht.

\subsection{Der bisherige Projektverlauf}

Die Faszination, die das Projekt ausübt, lässt sich bereits daran ablesen, dass es eine wachsende Gruppe von Projektpartnern und darüber hinaus eine Vielzahl weiterer Tagungsteilnehmer über einen inzwischen mehrjährigen Zeitraum beschäftigt hat. Erwähnung verdienen insoweit zunächst die in den Jahren 2001 und 2002 durchgeführten Konferenzen, deren Ergebnisse in jeweils einem Sammelband unter den Titeln „Teilhabe behinderter Menschen an der Bürgergesellschaft in Asien und Europa - Eingliederung im Sozial- und Rechtvergleich“ sowie „Behinderung in Asien und Europa im Politik- und Rechtsvergleich (mit einem Beitrag zu den USA)“ in den Jahren 2002 und 2003 veröffentlicht worden sind. Als Herausgeber fungierten die Herren von Maydell, Pitschas und Schulte, die dieses Projekt konzipiert und seither in enger Kooperation verfolgt haben. Auch in der Phase zwischen dem zweiten Band und dem nunmehr durchgeführten Workshop sind sie in Kontakt und dem Thema verbunden geblieben, wovon eine Reihe weiteren Arbeiten zeugen, die in engem Bezug hierzu stehen. ${ }^{1}$ Für den jüngsten Workshop sind zu den drei bisherigen

1 So hat etwa Schulte in der Zwischenzeit vergleichend zum eng mit dem Behindertenrecht verbundenen Betreuungsrecht gearbeitet, und zwar insbesondere auch unter Einbezug asiatischer Perspektiven, vgl. dazu Schulte, Betreuungsrecht und soziale Grundsicherung, BtPrax 2006, 210 ff., ders., Betreuung: Rechtsfürsorge im Sozialstaat aus sozialrechtlicher Perspektive, in: Zander (Hrsg.), Rechtsfürsorge im Sozialstaat: Was ist Aufgabe der Betreuung? Ergebnisse des 9. Vormundschaftsgerichtstags, Bochum 2005, S. 29 ff.; sowie schließlich den auf Japanisch publizierten Beitrag „Theoretische Aufgaben des deutschen Betreuungsrechts“, in: Betreuungsrecht (Japan), 
Organisatoren noch die Herren Pörtner und Zachmann hinzugekommen, um die kulturwissenschaftliche Perspektive in der Konzeption stärker zu betonen.

\subsection{Die Attraktivität des Projekts und ihre Gründe}

Die augenfällige Faszination, die von diesem Projekt ausgeht, erscheint bei näherem Hinsehen durchaus erklärlich. Vor allem zwei Gründe kommen hierfür in Betracht.

\subsubsection{Verschiedenheit der betrachteten Regionen}

Der erste Grund hierfür dürfte zunächst darin liegen, dass es derart unterschiedliche Regionen sind, die einander gegenübergestellt werden. Auf der einen Seite wird Europa häufig und wohl auch mit einiger Berechtigung als Wiege des modernen Sozialstaats apostrophiert - bei allen Problemen, die dessen Erhalt und Anpassung seit langem aufwerfen. ${ }^{2}$ In Asien auf der anderen Seite geht es demgegenüber um eine deutlich jüngere Entwicklung, die sich mitunter in Anlehnung oder Abgrenzung zu den europäischen Erfahrungen vollzogen, dabei aber durchaus neue Formen hervorgebracht hat - oder diese angesichts der erst allmählich durchgreifenden ökonomischen Entwicklung in manchen der in das Projekt einbezogenen Staaten auch erst hervorzubringen begonnen hat.

Diese Konstellation verspricht Erkenntnisgewinne gleich in mehrfacher Hinsicht. ${ }^{3}$ Nicht nur besteht die Aussicht, dass der Vergleich prospektiv eine - ggf. adaptierte Rezeption übertragbarer Politiken vorbereiten könnte. ${ }^{4}$ Vielmehr verheißt retrospek-

4/2003, S. 352 ff.; Erwähnung verdient des Weiteren die von Pitschas betreute vergleichende Dissertation von Won über die bildungs- und insbesondere schulpolitische Behandlung junger behinderter Menschen; vgl. schließlich ferner noch den im Max-Planck-Institut für ausländisches und internationales Sozialrecht entstandenen Band über Perspektiven der schulischen Integration von Kindern mit Behinderung - Vergleichende und interdisziplinäre Betrachtungen, Baden-Baden 2004 (herausgegeben von Becker/Graser).

2 Die verschiedenen Herausforderungen, denen sich der Wohlfahrtsstaat gegenübersieht, werden in unzähligen Publikationen thematisiert; vgl. statt vieler beispielsweise zu den Folgen immer durchlässiger werdender Grenzen, Leibfried/Pierson (Hrsg.), Standort Europa. Sozialpolitik zwischen Nationalstaat und Europäischer Integration, Frankfurt am Main 1995, zur gesellschaftlichen Alterung Reinhard (Hrsg.), Demographischer Wandel und Alterssicherung, Rentenpolitik in neun europäischen Ländern und den USA im Vergleich, Baden-Baden 2001; mit einem vergleichenden Überblick zum Kostenanstieg im Gesundheitswesen vgl. Henke/Schreyögg, Towards sustainable health care systems, Berlin 2004, S. 27 ff.; zum Wandel der Arbeitswelt schließlich Zacher, Der Wandel der Arbeit und der sozialen Sicherheit im internationalen Vergleich, ZIAS 1999, S. 1 ff.

3 Für eine ausführlichere Auseinandersetzung mit den Erkenntnismöglichkeiten der Rechtsvergleichung, auf der die vorliegende Darstellung aufbaut, vgl. Graser, Dezentrale Wohlfahrtsstaatlichkeit im föderalen Binnenmarkt?, Berlin 2001, S. $110 \mathrm{ff}$.

4 Als klassisch gilt insofern die Formulierung, dass der Rechtsvergleich der Anreicherung des „Vorrates an Lösungen“; vgl. dazu etwa Zweigert/Kötz, Einführung in die Rechtsvergleichung, 3. Aufla- 
tiv die Betrachtung vergangener Transfers solcher Politiken, der dabei erfolgten Anpassungen sowie der tatsächlich erzielten Wirkungen vielfältige Aufschlüsse über die Interaktion politischer Steuerung und sozialer Wirklichkeit. ${ }^{5}$ Und nicht zuletzt verspricht ferner die Gegenüberstellung der unterschiedlichen regionalen Entwicklungen in einer breiteren Makro ${ }^{6}$-Perspektive ein klareres Bild von den jeweiligen regionalen Charakteristika in diesem Politikfeld, das - bei aller Vorsicht - Rückschlüsse auf die institutionalisierten Werthaltungen und deren Kontrast erlaubt. ${ }^{7}$

\subsubsection{Behindertenrecht also Lackmustest der Sozialstaatlichkeit}

Ein weiterer Grund für die Faszination dürfte sein, dass mit der Behindertenpolitik ein Politikfeld gewählt wurde, in dem die humanitäre, menschenrechtliche Dimension noch stärker im Vordergrund steht als in den meisten anderen Bereichen der Sozialpolitik. Gewiss, auch in diesem Bereich können sich sozial- und wirtschaftspolitische Interessen einmal decken, kann Sozialpolitik, um es unverblümt zu fassen, sich „rechnen“, so wie es etwa von Studien- und Arbeitsförderung, ja in mancher Hinsicht auch von Gesundheits- und Rentenpolitik gesagt werden könnte. Man sollte diese Dimension schon deswegen nicht aus den Augen lassen, weil sie stets und auch im der Fall der Behindertenpolitik Möglichkeiten eröffnen könnte, die sonst nicht erreichbar wären. Doch bei allen Fortschritten, die ein in dieser Hinsicht geschärftes Bewusstsein der Behindertenpolitik bereits beschert haben mag oder noch zu bescheren verheißt, so wäre doch die Hoffnung, eine weitgehende Deckung ökonomischer und sozialpolitischer Interessen zu erreichen, in diesem Bereich wohl illusorischer noch als in den meisten anderen Feldern der Sozialpolitik.

Ähnliches gilt für die Möglichkeit, Regelungen in diesem Politikfeld als bloßes Produkt einer erfolgreichen Interessendurchsetzung durch die Betroffenen zu deuten. Wieder gilt, dass dies auch im Bereich der Behindertenpolitik keineswegs ausgeschlossen ist. So verzeichnet man vielerorts eine zunehmende Organisiertheit der Betroffenen $^{8}$ und hat es auch in diesem Politikfeld mit zuweilen schlagkräftigen Organisationen zu tun. Aber bei allem Respekt für diese Entwicklung wäre es meist doch irreführend, die entsprechenden Verbände mit Blick auf ihren Einfluss auch

ge Tübingen 1996, S. 14, sowie Rheinstein, Einführung in die Rechtsvergleichung, (herausgegeben von v. Borries), 2. Auflage München 1987, S. 26.

$5 \mathrm{Zu}$ diesem methodologischen Verständnis solcher Transferstudien - oder mit einem anderen Wort: der Rezeptionsforschung - als einer invertierten Form des Rechtsvergleichs siehe Hänlein, Sozialrechtsvergleichung als Rezeptionsforschung?, ZIAS 1998, S. $104 \mathrm{ff}$.

6 Eingehend zum Begriff des „Makrovergleichs“ Bartels, Methode und Gegenstand intersystemarer Rechtsvergleichung, Tübingen 1982, S. $127 \mathrm{f}$.

7 Prägend zu diesem Verständnis des Rechtsvergleichs als Kulturvergleich Häberle, Rechtsvergleichung im Kraftfeld des Verfassungsstaates, Berlin 1992; besonders deutlich beispielsweise auf S. 40 .

8 Insbesondere für China hat Ding $\mathrm{Na}$ diesen Befund hervorgehoben; mit einer entsprechenden Beobachtung auch bereits Darimont, Landesbericht China, in: v.Maydell/Pitschas/Schulte (Hrsg.), Behinderung in Asien und Europa im Politik- und Rechtsvergleich, S. 272 f. 
nur in die Nähe der großen Interessenorganisation im Bereich der Sozialpolitik zu rücken. Allein die quantitative Dimension dürfte, gerade wenn es um Menschen mit schweren Behinderungen geht, jeder solchen Analogie entgegenstehen. Umso mehr freilich sind vorgefundene Regulierungen eher humanitär denn interessenpolitisch zu deuten.

In diesem zweifachen Sinne also kann das Behindertenrecht als Lackmustest für die Existenz und Umsetzung sozialpolitischer Steuerungsambitionen - oder kurz: für Sozialstaatlichkeit ${ }^{9}$ - gelten. Es eignet sich mithin ideal für einen Vergleich, der nicht allein Regulierungstechniken, sondern auch die ihrer Wahl zugrunde liegenden Werthaltungen in den Blick nimmt.

\subsection{Zur Ambitioniertheit des Projekts}

So faszinierend das Projekt sein mag, und so groß seine Erkenntnispotenziale, so ambitioniert ist es auch. Wieder lässt sich dies an seinem bisherigen Verlauf ablesen: Die drei Tagungen wurden mit großem Aufwand vorbereitet, konzentriert durchgeführt und gründlich nachbereitet. Sie haben, davon zeugen die jeweiligen Publikationen, deutliche Fortschritte gebracht. Dennoch ist das Erkenntnispotenzial des Projekts noch nicht ausgeschöpft und könnte es in diesem Stadium auch noch nicht sein. Die Ursachen hierfür liegen jedenfalls im Rückblick auf der Hand: Verantwortlich sind die Vielzahl der einbezogenen Länder, die Verschiedenheit der Regionen, die Breite des Themas sowie schließlich auch dessen Komplexität.

\subsubsection{Vielzahl der einbezogenen Länder}

Was die Vielzahl angeht, so bedarf diese Feststellung kaum der weiteren Erläuterung. Zwar sind Mehrländervergleiche in den Sozialwissenschaften und insbesondere in den sozialwissenschaftlichen Politikanalysen keine Seltenheit. ${ }^{10}$ Etwas anderes gilt aber, wenn diese Vergleiche mit einer Tiefenschärfe angestellt werden, wie sie bei rechtsvergleichenden Ansätzen anzutreffen und insbesondere auch im vorliegenden Projekt angestrebt ist. Gemessen daran handelt es sich bereits aufgrund der schieren Zahl einbezogener Länder um ein breit angelegtes Unterfangen.

9 Umfassend zum Begriff bereits Zacher, Das soziale Staatsziel, § 25 in: Isensee/Kirchhof (Hrsg.), Handbuch des Staatsrechts, Heidelberg 1987.

10 Kritisch über die Erträge vieler solcher vergleichender Studien hat sich in jüngerer Zeit u.a. Marmor geäußert. Er attestiert dem (seinem!) Feld der "comparative policy studies.... considerable gap between promise and performance", und konstatiert darüber hinaus "an extraordinary imbalance between the magnitude and speed of the information flows and the capacity to learn useful lessons from them"; vgl. Marmor/Freeman/Okma, Comparative Perspectives and Policy Learning in the World of Health Care, Journal of Comparative Politics Vol. 7 (2005), S. 331. 


\subsubsection{Verschiedenheit der betrachteten Regionen}

Mehr noch als die Anzahl schlägt freilich die Verschiedenheit der betrachteten Systeme zu Buche. Zwischen Japan, Südkorea, Indien und China liegen buchstäblich Welten, im Hinblick auf die ökonomische Entwicklung, Rechts- und Verwaltungstraditionen sowie geistesgeschichtlich-kulturelle Wurzeln. Die auf europäischer Seite betrachteten Systeme mögen demgegenüber zwar relativ homogen erscheinen. Aber auch hier sind die Unterschiede erheblich, gerade wenn man bedenkt, dass sich der Kreis der bisher einbezogenen Systeme keineswegs auf die Kernstaaten des vereinten Europas beschränkt.

\subsubsection{Breite des Themas}

Ein weiterer, nicht minder bedeutsamer Aspekt ist in diesem Zusammenhang die Breite des betrachteten Politikfeldes. Mit dem Begriff der Behinderung ist ein Phänomen umschrieben, dessen Ursachen und Erscheinungsformen ebenso vielfältig sind wie die Handlungen oder Handlungsabsichten, die es auf Seiten der Akteure kollektiver (Selbst-)Steuerung auslöst. Das Spektrum möglicher Maßnahmen reicht von Geldleistungen zur Deckung der Grundbedarfe bis hin zum gesetzlich festgeschriebenen Erfordernis rollstuhlgerechter Zugänge für Neubauten, von der Organisation und Finanzierung ambulanter heilpädagogischer Dienste zur Förderung entwicklungsverzögerter Kinder bis hin zur Definition steuerlich absetzbarer Sonderbelastungen infolge einer Behinderung.

\subsubsection{Komplexität des Themas}

Man hat es also mit einer Vielfalt sozialer Problemlagen, möglicher Politikziele und Umsetzungsinstrumente zu tun, und entsprechend komplex ist die Materie, die im Projekttitel so scheinbar schlicht mit „Behindertenrecht und -politik“ bezeichnet ist. Pflege-, Kranken-, Mindestsicherungssysteme sind angesprochen. Regulierungen vom Arbeits-, über das Steuer-, bis hin zum Baurecht sind einschlägig. Man muss die Organisation von Kindergärten, Schulen, Werkstätten, Heimen, die Interaktion staatlicher und privater Akteure einschließlich der Zwischenformen studieren, und dazu typischer Weise auch das Zusammenspiel verschiedener politischer Ebenen einbeziehen, von der Gemeinde bis - gerade in Europa - hinein in den transnationalen Raum. 


\section{Der Workshop im Kontext der vorangegangenen Tagungen}

Sind das Potenzial des Projekts, sein Reiz und seine Herausforderungen damit immerhin grob nachgezeichnet, so soll im Folgenden eine Zwischenbilanz gezogen werden. Oder - direkt gefragt: Wie ist der Stand nach der dritten Tagung?

\subsection{Zielsetzungen des Workshops im Lichte der vorangegangenen Projektschritte}

Wie von Maydell in seinem Einführungsreferat zum Workshop ausführte, war es ein Verdienst bereits der ersten beiden Tagungen innerhalb des Projekts gewesen, die Vielfalt und Komplexität des Themas, wie sie im Vorangegangenen nur angedeutet werden konnte, zu erfassen und zu konturieren. Zunächst und vor allem war dies aus rechts- und verwaltungswissenschaftlicher Perspektive geschehen, wie es auch der disziplinären Ausrichtung der in diesem Stadium am Projekt beteiligten Personen entspricht. Zugleich waren mit der zunehmenden Differenziertheit dieser rechts- und verwaltungswissenschaftlichen Analysen aber auch deren zahlreiche Bezüge zu jenen Rahmenbedingungen zunehmend deutlich geworden, die dem juristisch oder verwaltungswissenschaftlich geschulten Blick nicht unmittelbar zugänglich sind.

Folgerichtig wurde die nächste Tagung - der Workshop, der im Zentrum der vorliegenden Betrachtungen steht - ganz dem Ziel gewidmet, diesen Bereich besser auszuleuchten. Es galt also, vom Boden der rechts- und verwaltungswissenschaftlichen Bestandsaufnahmen ausgehend, die soziale Ambiance ${ }^{11}$ der rechtlichinstitutionellen Strukturen näher zu erkunden. Man kann insofern von einem im weiten Sinne rechts- und verwaltungssoziologischen Erkenntnisinteresse sprechen und dem Projekt in dieser Hinsicht ein beachtliches Innovationspotenzial attestieren. Denn bei allem Zuspruch, den die Interdisziplinarität seit einiger Zeit erfährt, werden derartige Fragestellungen gegenwärtig dennoch allzu selten verfolgt. ${ }^{12}$

11 Zu diesem Begriff vgl. Häberle, Die Wesensgehaltsgarantie des Art. 19 Abs. 2 Grundgesetz, 3. Aufl. 1983, S. 415; auch schon Zacher, Vorfragen zu den Methoden der Sozialrechtsvergleichung, S. 21 ff., in: ders. (Hrsg.), Methodische Probleme des Sozialrechtsvergleiches, Colloquium der Projektgruppe für Internationales und Vergleichendes Sozialrecht der Max-Planck-Gesellschaft, Berlin 1977, der auf S. 61-66 zahlreiche Facetten dieser „Ambiance“ differenziert.

12 Eine Renaissance der Rechtssoziologie (und -anthropologie) im Gefolge der zunehmenden Pluralisierung positiver Rechtsordnungen verzeichnet in jüngerer Zeit Günther, Rechtspluralismus und universaler Code der Legalität: Globalisierung als rechtstheoretisches Problem, in: ders./Wingert (Hrsg.), Die Öffentlichkeit der Vernunft und die Vernunft der Öffentlichkeit, Festschrift für Jürgen Habermas, Frankfurt am Main 2001, S. 539 ff. Zugleich attestiert er dieser Disziplin bei dieser Gelegenheit zu Recht, seit langem ein Schattendasein geführt zu haben (S. 552). 


\subsection{Ausleuchten des „kulturwissenschaftlichen Hintergrundes“}

Der explorativen Zielsetzung des Workshops entsprechend, wurde weit ausgegriffen. Um eine „kulturwissenschaftliche Grundlegung und (um) Erklärungshypothesen" sollte es gehen. Dementsprechend reichte das Spektrum von Aspekten der Religions- und Geistesgeschichte ${ }^{13}$ über mikrosoziologische Betrachtungen der Bedingungen in Familie, ${ }^{14}$ Schule ${ }^{15}$ und am Arbeitsplatz ${ }^{16}$ bis hin zu Analysen der politischen Interessen- und Entscheidungskonstellationen ${ }^{17}$ im Bereich der Behindertenpolitik sowie deren ökonomischer Voraussetzungen. ${ }^{18}$

Bereits diese Aufzählung macht deutlich, dass es nicht um eine umfassende Würdigung all dieser Umstände und ihrer Relevanz für die rechts- und verwaltungswissenschaftlich erfassten Strukturen gehen konnte. Im Vordergrund stand stattdessen das Bestreben, anhand einzelner Betrachtungen exemplarisch zu plausibilisieren, welche Bedeutung diesen Aspekten im Kontext des Projekts zukommen kann. Diese Plausibilisierung ist ohne Frage gelungen, und zugleich wurde dabei eine Vielzahl von Impulsen für künftige Projektschritte gegeben.

So wurde beispielsweise in mehreren Beiträgen ${ }^{19}$ zur Situation in unterschiedlichen Regionen Asiens thematisiert, dass von Geburt an behinderte Menschen als Strafe für Eltern und Familie wahrgenommen würden. Dies ist ein Umstand, der die Rahmenbedingungen behindertenpolitischer Interventionen wesentlich beeinflusst und von den in Europa vorgefundenen absetzt.

Einen deutlichen Kontrast insbesondere mit Blick auf die politischen Zielsetzungen hat ferner der Bericht über die Förderschulentwicklung in Südkorea zu Tage gefördert. ${ }^{20}$ Während man in Europa gegenwärtig einen deutlichen Akzent auf integrative Beschulungsansätze legt, dominiert in der koreanischen Entwicklung klar die Idee einer institutionellen Separation. Auch wenn derlei Leitbilder gerade in einer historischen Perspektive keineswegs unveränderlich erscheinen - gerade in Deutschland liegt der Paradigmenwechsel in diesem Bereich noch nicht lange zurück $-{ }^{21}$ so müssen die hier hervortretenden Unterschiede doch berücksichtigt werden, wenn es um einen Vergleich der aktuellen Lage geht.

13 Außer im Referat von Pörtner und in den Diskussionsbeiträgen von Zachmann wurden diese Aspekte insbesondere auch in den Beiträgen von Gnanasekaran und Won thematisiert.

14 Vgl. dazu vor allem den Beitrag von Motozawa.

15 Vgl. dazu den Beitrag von Won.

16 Vor allem im Zusammenhang mit den Beiträgen über Korea und Japan wurden diese Aspekte diskutiert.

17 Eine besondere Betonung hat dieser Aspekt im Referat von van Langendonck erfahren.

18 Dieser Aspekt wurde insbesondere in Pitschas' Analyse der Interaktion mehrerer Verwaltungsebenen in Deutschland eingehend behandelt.

19 Das gilt namentlich für die Beiträge von Gnanasekaran, Won und Na.

20 Von Won zum Thema „Bildungspolitik für junge Menschen und die Entwicklung von Gesetzgebung und Rechtsprechung“.

21 Vgl. dazu jüngst Powell, Behinderung in der Schule, behindert durch Schule? Wie „schulische Behinderung" institutionalisiert wurde, in: Waldschmidt/Schneider (Hrsg.), Disability Studies, Kultursoziologie und Soziologie der Behinderung, Bielefeld 2007, S. $321 \mathrm{ff}$. 
Ebenfalls die politischen Zielsetzungen und Problemdefinitionen betreffend, hat Pörtner in seinem einleitenden Beitrag vorgeführt, dass nicht einmal die grundlegende Prämisse des Projektes unhinterfragt bleiben könne. Gemeint ist die implizite Annahme, dass die Verbesserung der Situation behinderter Menschen Gegenstand und Auftrag kollektiver Selbststeuerung - „der Politik“ mit anderen Worten - zu sein habe. Offensichtlich ist dieser Aspekt für das gesamte Projekt von fundamentaler Bedeutung. Zwar haben die neueren internationalrechtlichen Vorgaben, ${ }^{22}$ wie sie insbesondere in der Referaten von Trenk-Hinterberger und Schulte erläutert wurden, eine gewisse Universalisierung dieser womöglich westlich-europäischen Prämisse gebracht, auf deren Grundlage die ,globalere“ Ausrichtung des vorliegenden Projektes jedenfalls berechtigt erscheint. Zumal aber Konkretion und Bindungsgrad solcher internationalrechtlicher Zielvorgaben bekanntlich gering und damit die Räume für unterschiedliche nationale Ausprägungen groß sind, bleibt der Hinweis von Pörtner dennoch gültig und sollte die weiteren Schritte des Projekts im Sinne eines, wie er es selbst formuliert hat, „mitlaufenden Zweifels“ begleiten.

\subsection{Brückenbau ausgehend vom Boden der Rechts- und Verwaltungswissenschaft}

Parallel und gleichsam als Gegenstück zu den primär gesellschafts- oder kulturwissenschaftlich orientierten Beobachtungen gab es eine Reihe von Beiträgen, die ihren Ausgangspunkt erneut in den Rechts- oder Verwaltungswissenschaften genommen haben, um aber von dort dann mit dem Bau von Brücken zu jenen Umständen außerhalb ihrer genuinen disziplinären Zuständigkeit zu beginnen. Wieder war das Spektrum breit.

\subsubsection{Vom materiellen Recht ausgehende Erwägungen}

Ausgehend von den Darstellungen zum materiellen Recht wurde beispielsweise wiederholt erörtert, welche Leitbilder ${ }^{23}$ den jeweils betrachteten behindertenpolitischen Maßnahmen zugrunde lägen - von Fürsorge über Gleichstellung bis hin zu Inklusion - und inwiefern sie unter den jeweiligen Bedingungen adäquat und für die verschiedenen Gruppen von Betroffenen interessengerecht seien. Dabei wurde nicht nur ein breites Arsenal rechtlicher Instrumente sichtbar, das der gesetzgeberischen Inspiration und so einem klassischen Ziel der Rechtsvergleichung dienen könnte. ${ }^{24}$ Vielmehr wurde auch die Kontextgebundenheit dieser Instrumente deutlich, deretwegen simple Übertragungsversuche von vornherein ausscheiden. Besonders einge-

22 Namentlich handelt es sich um die UN Convention on the Rights of Persons with Disabilities aus dem Jahr 2006; für nähere Angaben vgl. insbesondere das Referat von Schulte.

23 Besonders prononciert hat solche Leitbilder insbesondere Trenk-Hinterberger in seinem Beitrag über „Leistungen für behinderte Menschen in Europa“ herausgearbeitet.

Vgl. dazu oben Fn. 3-7 sowie den zugehörigen Text. 
hend behandelt wurde dies in Cheons Bericht über die Entwicklung des südkoreanischen Behindertenrechts, die gekennzeichnet ist von einer raschen, aber doch kontextsensiblen und mithin adaptierenden Rezeption westlicher Regelungsansätze.

Ebenfalls aus den Erörterungen des materiellen Rechts ergaben sich wiederholt aufschlussreiche Perspektiven auf die Interdependenz juristischer Kategorisierungen und politischer Interessenformationen. ${ }^{25}$ Auffällig ist dies beispielsweise im Hinblick auf den Behindertenbegriff. Ein breiter und umfassender Zuschnitt der entsprechenden gesetzlichen Begriffe könnte insofern zur Formation großer und zunächst ähnlich interessierter Gruppen führen und diesen mehr Durchsetzungskraft verleihen. Als ein einschlägiges Beispiel wurden in der Diskussion die USA erwähnt. ${ }^{26}$ Dagegen trifft man nicht selten auf eher enge Definitionen. Beispielsweise lässt sich wohl nur so erklären, warum man in Indien von einer insgesamt soviel geringen Zahl behinderter Menschen ausgeht. Hinzu kommt, dass die Grenzziehungen, wie sie gerade in stark ausdifferenzierten Systemen des sozialen Schutzes unvermeidbar sind, mitunter diffizil sind und ähnliche Interessenlagen künstlich auseinanderdividieren könnten. Als Beispiel mag in Deutschland etwa die Abgrenzung zwischen Rehabilitation und Pflege dienen.

\subsubsection{Vom prozeduralen Recht ausgehende Erwägungen}

Viel Aufmerksamkeit wurde ferner den sogenannten „enforcement“-Fragen zuteil, also dem Aspekt der effektiven Durchsetzung von (hier: behinderten-) politischen Zielen und Regelungen. Dabei kann es sich um Durchsetzung mittels sowohl exekutiver als auch judikativer Mechanismen handeln, und wie zuvor wurde auch hier das Wechselspiel dieser institutionellen Strukturen mit den im weiten Sinne sozialen Rahmenbedingungen augenfällig.

Im Einzelnen ging es auch in diesem Themenfeld zunächst erneut darum, aus der vergleichenden Betrachtung ein Arsenal unterschiedlicher Instrumente einschließlich der Erfahrungen mit ihrem praktischen Einsatz zusammenzutragen. Eingehend wurden erstens die verschiedenen Wege administrativer Rechtsdurchsetzung erörtert, die von kommunalen oder betrieblichen Behindertenbeauftragen über weniger dezentral angesiedelte Ombudspersonen bis hin zu Behörden reichen, die eigens mit der Überwachung oder gar Durchsetzung der entsprechenden Normen befasst sind. Insbesondere Köhlers Analyse der entsprechenden Strukturen in Schweden hat diese Perspektive in den Vordergrund gerückt.

Allgemeiner zu diesem Konnex Graser, Zur Fragmentierung der Mindestsicherung - Eine Hypothese zum Zusammenhang zwischen dem Geltungsbereich eines sozialrechtlichen Regelwerks und seinem materiellen Schutzgehalt, ZIAS 2003, S. $319 \mathrm{ff}$.

26 Näher zum breiten Behindertenbegriff in den USA und zum entsprechend hohen Anteil der Behinderten an der dortigen Bevölkerung, Graser, ebda., S. 320 f. sowie ders., Landesbericht USA, in: v.Maydell/Pitschas/Schulte (Hrsg.), Behinderung in Asien und Europa im Politik- und Rechtsvergleich, S. 234. 
Im Hinblick auf die Formen der klassischen, also judikativen (Rechts-)Durchsetzung wurde zweitens vor allem über die Beschränkungen gesprochen, wie sie mit der typischer Weise retrospektiven und auf den Einzelfall beschränkten Ausrichtung dieser Verfahren einhergehen. Als alternative Optionen stehen class actions oder Verbandsklagen zur Diskussion. Freilich handelt es sich auch dabei um Instrumente, die nicht unbesehen in einen neuen Kontext importiert werden sollten.

Mit dem Verweis auf die Kontextabhängigkeit dieser Instrumente ist drittens ein weiterer Ertrag angesprochen, den die Betrachtungen der enforcement-Fragen gebracht haben. Denn auch in diesem Zusammenhang wurde deutlich, wie sehr die Effektivität der rechtlichen und Verwaltungsstrukturen anhängt von in weiterem Sinne sozialen Parametern. So läuft etwa das Instrument der Verbandsklage offensichtlich leer, solange es an einer hinreichend organisierten Verbandsstruktur fehlt. Grundlegender noch wurde mit Blick auf alle Instrumente, die einen subjektivrechtliche Komponente enthalten, wiederholt die These aufgestellt, dass deren gerichtliche Rechtsverfolgung in manchen asiatischen Ländern deutlich weniger üblich sei als in den westlichen und insbesondere auch den europäischen Kulturen. Gewiss bedürfte diese Behauptung noch einer empirischen Fundierung speziell für den gegebenen Kontext des Behindertenrechts. Fest steht aber, dass dieser Unterschied für einen eventuellen Transfer von Regelungsinstrumenten aus oder nach Europa offensichtlich von erheblicher Bedeutung wäre.

\subsubsection{Verwaltungsstrukturen und Zivilgesellschaft}

Die soeben erwähnten Verbandsstrukturen sind freilich nicht nur aus prozessrechtlicher, sondern auch aus verwaltungswissenschaftlicher Perspektive von zentraler Bedeutung. Diese - oft mit dem schillernden Begriff der Zivilgesellschaft umschriebenen - Strukturen „Zwischen Staat und Markt“, wie sie im (leider entfallenen) Referat von Lachwitz bezeichnet sind, sind eine entscheidende Determinante für die konkrete Gestalt der behindertenpolitischen Verwaltungsorganisation - und darüber hinaus auch für die Möglichkeiten der Behindertenpolitik insgesamt. Ein Blick auf die Rolle, welche die freien Träger in Deutschland für die Erbringung behindertenpolitischer Leistungen spielen, verdeutlicht dies eindrucksvoll. ${ }^{27}$

Wenn es darum geht, Behindertenrecht und -politik zu vergleichen und insbesondere mit Blick auf ihre Gestaltungsmöglichkeiten zu erfassen, kommt man nicht umhin, diese zivilgesellschaftlichen Strukturen und ihre vielfältigen Verschränkungen mit den unterschiedlichen Ebenen staatlicher Verwaltung einzubeziehen. Dieser Blickwinkel der - mit einem Modewort - ,governance“-Forschung ist für die Behin-

27 Für eine systematische Auseindersetzung mit diesen „privaten“ Erscheinungsformen unter dem Blickwinkel ihrer Beziehung zum Staat siehe Geis, Die öffentliche Förderung sozialer Selbsthilfe Verfassungsrechtliche Grundlagen und verwaltungsrechtliche Ausgestaltung, Baden-Baden 1997. 
dertenpolitik unumgänglich. Insbesondere Pitschas hat diese Aspekte in seinem Referat ${ }^{28}$ wie auch im Rahmen der Diskussionen wiederholt eingebracht.

\subsection{Demographischer Wandel als Querschnittsthema}

Besondere Berücksichtigung erfuhr im Rahmen des Workshops ferner der Aspekt des demographischen Wandels - teils in Form eines Querschnittsthemas, das sämtliche Referate berührte und dementsprechend in unterschiedlichen Zusammenhängen immer wieder zur Sprache kam; teils aber auch im Rahmen des eigens hierauf fokussierenden Beitrags von Kruse. Danach ist der demographische Wandel ist für die Behindertenpolitik vor allem in zweierlei Hinsicht relevant.

\subsubsection{Zunahme von Behinderungen aufgrund steigender Lebenserwartung}

Zum einen ist im Zuge einer steigenden Lebenserwartung damit zu rechnen, dass das Phänomen altersbedingter Behinderungen zunehmen wird. Das kann jedenfalls dann gelten, wenn dieser Zuwachs auf einer Verlängerung einer dem Lebensende vorangehenden Phase wahrscheinlicher Gebrechlichkeit beruht. Für diese Annahme spricht der Umstand, dass die Lebenserwartung gerade in den Industriestaaten unter anderem aufgrund einer Ausdehnung der Phase des hohen Alters steigt. ${ }^{29}$ Allerdings bedürfte diese Annahme weiterer empirischer Erhärtung. Dabei kommt dem rechtlichen Begriff der Behinderung und den damit einhergehenden Abgrenzungsproblemen offensichtlich gesteigerte Bedeutung zu. Hier eröffnet sich eine Perspektive für künftige Forschung an der Schnittestelle von empirischer Soziologie und insbesondere demographischer Forschung auf der einen Seite und Rechtswissenschaft auf der anderen.

Ein Konnex zwischen steigender Lebenserwartung und der relativen Zunahme von Behinderungen kann ferner auch noch auf andere Entwicklungen zurückzuführen sein, die nicht allein die Phase des hohen Alters betreffen. So könnten medizinische Fortschritte etwa im Bereich der Versorgung Frühgeborener nicht nur die Lebenserwartung, sondern auch die relative Häufigkeit von Behinderungen steigern. Ähnliches könnte - mutatis mutandis - auch für Behandlungsmöglichkeiten in anderen Lebensphasen gelten. Abermals kann zu diesem Aspekt nur auf die Notwendigkeit einer weiteren Erforschung dieses relativ wenig behandelten Gebiets verwiesen werden, und insbesondere auf die Notwendigkeit (einer Rezeption) von demographi-

28 Zum Thema „Behinderte Menschen in der kommunalen Sozialpolitik“.

29 Einen weiteren Rückgang der Sterblichkeit in hohem Alter verzeichnen in jüngerer Zeit Rau/Soroko/Jasilionis/Vaupel: 10 years after Kannisto: further evidence for mortality decline at advanced ages in developed countries. MPIDR working paper WP-2006-033, Rostock 2006, im Internet verfügbar unter: http://www.demogr.mpg.de/papers/working/wp-2006-033.pdf 
schen Studien speziell zu den in diesem Projekt betrachteten Regionen und Fragestellungen.

\subsubsection{Steigerung der Lebenserwartung für Menschen mit Behinderung}

Noch ein weiterer, seltener thematisierter Konnex zwischen demographischer Entwicklung und Behinderung wurde in dem Referat von Kruse mit großer Eindringlichkeit unterstrichen. Kruse bezog sich auf neuere Studien, welche die Vermutung nahelegen, dass die Lebenserwartung von Menschen mit Behinderung wesentlich gesteigert werden könne, wenn sie auch nach der Phase der Berufsqualifikation und mithin in höherem Alter noch systematisch gefördert werden.

In einer humanitären Perspektive erfordern diese Befunde dringend ein Umdenken in der Gestaltung speziell von Heimumgebungen, aber auch des breiteren Angebotsspektrums der einschlägigen Sozialleistungssysteme. Diese Erkenntnisse betreffen, wie Kruse betonte, zunächst einmal Deutschland, dürften aber generalisierbar sein und könnten mithin maßgebend für eine entsprechende Rechtsentwicklung auch in anderen Regionen werden. Es wäre lohnende Aufgabe weiterer Forschung in diesem Bereich, die konkreten Erfordernisse und Möglichkeiten solcher eventuell gebotenen Anpassungen der Rechts- und Verwaltungsstrukturen in den verschiedenen Betrachtungslängern näher zu eruieren.

\section{4. Überlegungen zur Fortsetzung des Projekts}

Die vorangegangenen Erörterungen mögen jenen Befund untermauert haben, der vorab bereits angedeutet worden war: So ertragreich die bisherigen Tagungen einschließlich des hier referierten Workshops auch gewesen sein mögen, die Erkenntnispotentiale des Projekts sind noch nicht ausgeschöpft. Zuvor wurden mehrfach einzelne Forschungsfragen benannt, die sich aus den bisherigen Arbeiten ergeben haben und künftig weiter verfolgt werden könnten. In diesem letzten Abschnitt soll nun eine breitere Perspektive eingenommen und gefragt werden, wie das Projekt insgesamt auf Grundlage der bisherigen Erkenntnisse fortgeführt werden könnte.

\subsection{Bisherige Erträge}

Ohne die bisherigen Erfolge im Geringsten zu schmälern, lässt sich festhalten, dass die beiden großen Hoffnungen, die diesem Ansatz zu Anfang des vorliegenden Resümees zugeschrieben wurden, noch nicht vollends eingelöst sind. Denn weder lassen sich auf Ebene einer Makrobetrachtung der verglichenen Regionen gesicherte Aussagen treffen über divergierende Werthaltungen und deren Manifestationen im Recht der Menschen mit Behinderung. Noch könnten in einer Mikroperspektive auf 
Grundlage der bisherigen Erkenntnisse fundierte Empfehlungen zur Übertragung einzelner Maßnahmen von einer Region in die andere getroffen werden.

Gewiss wurden in beiderlei Hinsicht erhebliche Fortschritte erzielt. So konnte erstens das Arsenal denkbarer Regelungsinstrumente in sowohl materieller als auch prozeduraler als auch institutioneller Hinsicht angereichert werden, und zwar in einem Ausmaß, wie es wohl nur in einem derart ausgreifenden Vergleich möglich ist. Zweitens sind auch die Hypothesen über das Bestehen und die Relevanz erheblicher - im weiten Sinne: - „kultureller“ Unterschiede zwischen den betrachteten Regionen konkretisiert und erhärtet worden. Dem insbesondere sollte der Workshop ja dienen.

Und noch in einer dritten Hinsicht, die gleichsam dazwischen liegt, wurden wesentliche Fortschritte erzielt, nämlich beim Bemühen um einen Brückenbau zwischen diesen beiden Perspektiven, bei der Identifikation der konkreten Rahmenbedingungen also, die für das Funktionieren von Recht und Verwaltung maßgeblich sind. Vielleicht sind dies die wichtigsten Erträge. Jedenfalls wäre dies nicht untypisch für rechts- und wohl auch verwaltungsvergleichende Unternehmungen, wie das vorliegende Projekt eine ist. Diesseits von weitreichenden kulturwissenschaftlichen Erklärungsansätzen und praktisch verwertbarem Lösungsimport schärft sich im Wege eines solchen Vergleichs das Verständnis der mannigfaltigen Wechselbezüge von Rechts- und Verwaltungsstrukturen einerseits und ihrer sozialen Umgebung andererseits. In diesem Sinne war eingangs von einem rechts- und verwaltungssoziologischen Erkenntnisinteresse die Rede.

\subsection{Ausrichtung weiterer Schritte}

Freilich sollten die beachtlichen Erträge, die das Projekt bislang erbracht hat, nicht als Grund dafür dienen, weitere Schritte zu unterlassen. Dafür haben sich allzu viele fruchtbare Perspektiven eröffnet während der bisherigen, nicht umsonst durchwegs als Explorationen konzipierten Tagungen. Und auch eine mögliche Unerreichbarkeit der zuvor als „große Hoffnungen“ apostrophierten Maximalziele der Vergleichung sollte keineswegs zur Grundlage für den Abbruch der hierauf gerichteten Bemühungen werden. Im Gegenteil ist es gerade die systematische Verfolgung dieser Ziele und idealiter beider zusammen -, welche den Gewinn jedenfalls der recht- und verwaltungssoziologischen Verständnisschärfung ermöglicht.

Wenn das Projekt demnach fortgeführt werden sollte, so gilt es zum Abschluss noch zu erörtern, wie es weitergehen könnte. An Anknüpfungspunkten - vor allem dies sollte zuvor illustriert werden - besteht kein Mangel, und eine Auswahl daraus zu treffen, überstiege die Bestimmung des vorliegenden Beitrags. Deswegen sollen hier nur zwei Prinzipien angedeutet werden, die bei den Überlegungen über den Zuschnitt der nächsten Projektschritte vielleicht Berücksichtigung finden könnten. 


\subsubsection{Ausgleich bestehender Asymmetrien}

Der bisherige Projektverlauf ist von einer doppelten Asymmetrie gekennzeichnet: einer disziplinären und einer regionalen. Es hätte anders auch kaum sein können, muss doch die Annäherung an ein solches Groß-Projekt irgendwo ihren Ausgangspunkt nehmen, um von dort aus dann allmählich das gesamte Feld zu erschließen.

Beim vorliegenden Projekt war dieser Ausgangspunkt im diszisplinären Sinne die Rechts- und Verwaltungswissenschaft. Dies wurde zuvor im Einzelnen dargelegt, einschließlich der Schritte, die bereits unternommen worden sind, um diese anfängliche Beschränkung zu transzendieren.

In regionaler Hinsicht lag der Schwerpunkt bisher auf der Betrachtung Europas. Dabei handelt es sich um eine Implikation weniger des ursprünglichen Projektdesigns, das von vornherein in zumindest gleicher Weise auf asiatische Länder fokussiert war. Vielmehr dürfte diese regionale Asymmetrie bislang schlicht der Lokalität der bisherigen Tagungen geschuldet sein, die allesamt in Deutschland stattfanden. Im Ergebnis freilich ist dadurch die Detailschärfe der auf Europa und insbesondere Deutschland bezogenen Betrachtungen insgesamt deutlich höher ausgefallen als bei ihren asiatischen Pendants.

Für die nächsten Schritte erscheint es angezeigt, diese Asymmetrien auszubalancieren. In regionaler Hinsicht wird man diesem - übrigens allseits konsentierten Petitum bereits durch die Wahl des nächsten Tagungsorts Genüge tun können. In disziplinärer Hinsicht dagegen handelt es sich um eine anspruchsvollere Aufgabe. Denn um jene „soziale Ambiance“, deren systematische Erkundung auf dem hier resümierten Workshop erst begonnen hat, weiter zu erforschen, wird man die verschiedenen einschlägigen Zweige der Sozial- und Kulturwissenschaften noch deutlich weiter verfolgen müssen.

Dass dies der Mühe wert ist, hat der Workshop demonstriert. Zugleich hat er hierfür Anknüpfungspunkte geliefert und auch die Richtungen angezeigt, in welche die künftigen Forschungsfragen weisen sollten. Die größte Herausforderung könnte demnach darin bestehen, Experten aus den jeweiligen Disziplinen und Regionen zu finden, die diese speziellen Fragen behandeln. Bekanntlich kommt es nicht selten vor, dass jene Klärungsbedarfe, die Rechts- oder Verwaltungswissenschaft mit Blick auf die übrigen Sozialwissenschaften formulieren, nicht beantwortet werden (können). Es ist insofern absehbar, dass man sich bei der Bestimmung künftiger Arbeitsschwerpunkte auch an der Verfügbarkeit entsprechender sozialwissenschaftlicher Expertise wird orientieren müssen.

\subsubsection{Exemplarische Fokussierung}

Mit der Erwähnung künftiger Arbeitsschwerpunkte ist auch der zweite Orientierungspunkt für die künftige Projektplanung bereits angesprochen. Eines der größten Verdienste der bisherigen Arbeitsschritte innerhalb des Projektes war es, dass die Breite und Komplexität des Themas erfasst und konturiert worden sind - zunächst, 
wie gesehen, mit Blick vor allem auf die Rechts- und Verwaltungswissenschaft, im dritten Schritt nun auch zunehmend in einer im weiten Sinne soziologischen oder kulturwissenschaftlichen Perspektive. Wenn dabei aber zugleich wiederholt auch das Bedürfnis nach weiterer Vertiefung augenfällig geworden ist, so versteht es sich nahezu von selbst, dass man derartige Vertiefungen aus praktischen Gründen nicht auf der gesamten Breite des über drei Tagungen hinweg immer weiter entfalteten Themas wird leisten können.

Bei den nun anstehenden Vertiefungen wird man daher nach einzelnen, enger definierten Betrachtungsgegenständen Ausschau halten müssen. Das bedeutet nicht, dass damit die bisher angestrebte Gesamtperspektive und die damit verbundenen Erklärungsambitionen aufgegeben werden müssten. Das Ziel sollte vielmehr sein, die Gegenstände möglicher Vertiefungen so zu wählen, dass die dabei gewonnenen Erkenntnisse einer Verallgemeinerung zugänglich sein könnten.

Welche Fragestellungen sich für solche exemplarischen Vertiefungen besonders eigenen, kann man und haben auch die Tagungsteilnehmer im Rahmen der Schlussdiskussion unterschiedlich beurteilt. In Frage käme beispielsweise, anknüpfend an unterschiedliche Behinderungsursachen vertiefende Betrachtungen anzustellen, also etwa von Geburt an bestehende Behinderungen einerseits und alterungsbedingte andererseits näher in den Blick zu nehmen. Dafür spräche nicht nur, dass sich die vermuteten kulturellen Unterschiede im Umgang mit Behinderten am Gegenstand „angeborener“ Behinderungen wahrscheinlich besonders deutlich manifestieren würden. Auch und vor allem könnte man bei dieser Einteilung mit dem Schwerpunkt auf alterungsbedingte Behinderungen ein Thema weiter verfolgen, dessen regionenübergreifende Brisanz der hier resümierte Workshop besonders unterstrichen hat.

Einer anderen Logik folgend, könnten bei den anstehenden Vertiefungen unterschiedliche Lebensphasen in den Blick genommen werden, also etwa Ausbildung, Erwerbsleben und die Phase danach. Diese Einteilung hätte zum einen für sich, dass die bestehenden Maßnahmen der Behindertenpolitik zum Teil ähnlich gegliedert sind, was die Aufbereitung erleichtern würde. Zum anderen wäre es auch bei diesem Zugriff wieder möglich, dem Konnex von demographischem Wandel und Behinderung weiter nachzugehen, und zwar beiden Facetten, die zuvor im Zusammenhang mit dem Beitrag von Kruse dargestellten wurden.

Eine Festlegung hinsichtlich der Gegenstände vertiefter Betrachtungen liegt, wie gesagt, jenseits der Zielsetzungen dieses Beitrags. Zumal bei einer solchen Festlegung neben systematischen ferner auch pragmatische Erwägungen eine wichtige Rolle spielen werden, dürften allzu enge Festlegungen im gegenwärtigen Stadium auch noch nicht tunlich sein. Stattdessen liegt es nahe, bis auf Weiteres auf Grundlage einer Sammlung möglicher Schwerpunktthemen zu operieren, zu der die hier genannten zählen, aber durchaus auch noch weitere hinzu treten könnten. 